\title{
Hypofractionated radiotherapy for early glottic cancer: a retrospective interim analysis of a single institution
}

\author{
Jeong Won Lee 1 , Jeong Eun Lee ${ }^{2}$, Junhee Park², Jin Ho Sohn ${ }^{3}$, Dongbin Ahn ${ }^{3}$ \\ 'Department of Radiation Oncology, Catholic University of Daegu, School of Medicine, Daegu, Korea \\ ${ }^{2}$ Department of Radiation Oncology, School of Medicine, Kyungpook National University, Daegu, Korea \\ ${ }^{3}$ Department of Otorhinolaryngology-Head and Neck Surgery, School of Medicine, Kyungpook National University, Daegu, Korea
}

Purpose: To evaluate the results of hypofractionated radiotherapy (HFX) for early glottic cancer.

Materials and Methods: Eighty-five patients with cT1-2NOMO squamous cell carcinoma of the glottis who had undergone HFX, performed using intensity-modulated radiotherapy (IMRT, $n=66$ ) and three-dimensional conformal radiotherapy (3D CRT, $n$ $=19$ ) were analyzed. For all patients, radiotherapy was administered at $60.75 \mathrm{~Gy}$ in 27 fractions. Forty-three patients received a simultaneous integrated boost (SIB) of 2.3-2.5 Gy per tumor fraction.

Results: The median follow-up duration was 29.9 months (range, 5.5 to 76.5 months). All patients achieved complete remission at a median of 50 days after the end of radiotherapy (range, 14 to 206 days). The 5-year rates for locoregional recurrence-free survival was $88.1 \%$, and the 5 -year overall survival rate was $86.2 \%$. T2 stage was a prognostic factor for locoregional recurrencefree survival after radiotherapy $(p=0.002)$. SIB for the tumor did not affect disease control and survival $(p=0.191$ and $p=0.387$, respectively). No patients experienced acute or chronic toxicities of $\geq$ grade 3 . IMRT significantly decreased the dose administered to the carotid artery as opposed to 3D CRT $\left(V_{351} p<0.001 ; V_{501} p<0.001\right)$.

Conclusions: Patients treated with HFX achieved acceptable locoregional disease control rates and overall survival rates compared with previous HFX studies. A fraction size of 2.25 Gy provided good disease control regardless of SIB administration.

Keywords: Radiotherapy, Glottis, Radiation dose hypofractionation, Intensity-modulated radiotherapy

\section{Introduction}

Patients with T1-2NO early glottic cancer (EGC) have shown excellent local control and survival rates after undergoing either radiotherapy $(\mathrm{RT})$ or surgical treatment [1]. The probability of lymph node metastasis is low in EGC; thus, localized RT to the larynx can successfully treat EGC with voice quality preservation and low toxicity levels [2]. RT is therefore the preferred treatment option among these patients $[1,3]$. Several studies have investigated the importance of prognostic factors that can affect RT outcomes in patients with EGC, such as fraction size, total dose, and overall treatment time [4-6].

Some investigators have attempted to identify altered fractionated schedules. Better results have been reported for hypofractionated radiotherapy (HFX; a fraction size of more than $2.0 \mathrm{~Gy}$ ) than for conventional fractionated radiotherapy

Received 04 March 2019, Revised 30 May 2019, Accepted 17 June 2019.

Correspondence: Jeong Eun Lee, Department of Radiation Oncology, Kyungpook National University Hospital, 130 Dongdeok-ro, Jung-gu, Daegu 700-721, Korea. Tel: +82-53-420-5353, Fax: +82-53-426-3303, E-mail: jelee@knu.ac.kr (http:// orcid.org/0000-0002-2409-537X)

(C) This is an Open Access article distributed under the terms of the Creative Commons Attribution Non-Commercial License (http://creativecommons.org/ licenses/by-nc/4.0/) which permits unrestricted non-commercial use, distribution, and reproduction in any medium, provided the original work is properly cited.

www.e-roj.org 
(CFX; a fraction size of 1.8-2 Gy) [7-11]. However, the optimal fraction size, total dose, and treatment schedule are still unclear; furthermore, there is a lack of sufficient data regarding treatment of EGC by HFX performed using advanced techniques such as intensity-modulated radiotherapy (IMRT) and/or simultaneous integrated boost (SIB) technique [12]. Therefore, we analyzed the clinical and dosimetric outcomes of patients with EGC who received HFX with or without SIB technique at our institution.

\section{Materials and Methods}

\section{Patients}

We analyzed 85 patients with EGC who were treated with hypofractionated RT between January 2011 and December 2016. The inclusion criteria were as follows: (1) histologically and clinically confirmed EGC (cT1-2NOMO); (2) RT as a curative treatment; (3) availability to undergo laryngoscopy during RT and follow-up; (4) no prior RT administered to the neck. The stage of cancer was determined according to the 7th edition of the American Joint Committee on Cancer. Histologic characteristics and stromal invasion of tumor were confirmed via biopsy. This study was approved by the Institutional Review Board of Kyungpook National University Hospital (No. KNUH2017-09-021-001) and Kyungpook National University Chilgok Hospital (No. KNUCH2017-10-010).

\section{Radiotherapy}

Patients were immobilized in the supine position with a thermoplastic mask. For planning computed tomography (CT)scan, slice thickness of $3 \mathrm{~mm}$ was used. For patients who received three-dimensional conformal radiotherapy (3D CRT), the clinical target volume (CTV1) included the whole larynx from the inferior margin of the hyoid bone to the cricoid cartilage, and the planning target volume (PTV1) was defined as CTV1 $+1 \mathrm{~cm}$. For IMRT plan, CTV1 and PTV1 were the same volumes as those of 3D CRT. Among 66 patients receiving IMRT, SIB was performed in 43 patients, most of whom (76.7\%) had at least one of the factors including anterior commissure involvement, stromal invasion, or T2 stage. An additional planning target volume (PTV2) for SIB was generated by extending $0.5 \mathrm{~cm}$ from CTV2, which was expanded by $0.5 \mathrm{~cm}$ from the tumor.

In this study, the prescription dose to the PTV of the whole larynx (PTV1) was 60.75 Gy in 27 fractions of 2.25 Gy in all cases. In the patients receiving IMRT with $\mathrm{SIB}$, radiation doses of 2.25 Gy for PTV1 and 2.3-2.5 Gy for PTV2 per fraction were administered with the aim of tumor dose escalation. The carotid arteries were extended $1.5 \mathrm{~cm}$ craniocaudally to the PTV1. A 3D CRT was administered using two opposite parallel fields with or without a paired wedge and by modulating the field weight or performing dose normalization to improve target coverage. In IMRT, nine beam angles of $280^{\circ}-80^{\circ}$ or $250^{\circ}-110^{\circ}$ were used, and the beam numbers, directions, and weights were adapted to avoid the spinal cord and enhance conformity and homogeneity of dose distribution. IMRT plans were normalized such that the PTV of the whole larynx (PTV1) received $>95 \%$ of the prescription dose without an intention of carotid artery sparing. SIB plan was calculated by adding the PTV1 and the dose difference between the PTV1 and PTV2.

\section{Evaluation of treatment outcome and toxicity}

Patients were regularly evaluated by performing laryngoscopy and physical examinations during the treatment period and at routine follow-up visits. Post-treatment imaging studies included a CT scan at 2 months after RT and a positron emission tomography-CT scan at 3 months after RT. Acute and late toxicities were graded using the National Cancer Institute Common Toxicity Criteria version 4.0 from the start of RT. Furthermore, we analyzed dose-volume data, such as $\mathrm{V}_{356 \mathrm{y}}$ and $V_{50 G y}$ for the carotid arteries to predict complications caused by radiation exposure to the carotid arteries [13].

\section{Statistical analyses}

The primary endpoints were locoregional recurrence-free survival (LRFS). The secondary endpoints were overall survival (OS) and HFX toxicity. LRFS was defined as the time from the date of the end of RT to the date of the diagnosis of local and/ or regional disease failure. OS was defined as the duration between the date of the end of RT and the date of death from any cause. Disease control and survival rates were calculated using the Kaplan-Meier method and were compared using the log-rank test. A chi-square or Fisher exact test was used to compare the categorical variables between the treatment groups with or without SIB. We performed univariate and multivariate Cox proportional hazards analyses to evaluate the effect of variables on LRFS and OS. Mann-Whitney $U$ test was used to compare the difference in carotid artery doses between 3D CRT and IMRT. The biologically effective dose (BED) was calculated according to the linear-quadratic model.

\section{Results}

Patient and tumor characteristics are listed in Table 1. The 
Table 1. Patients and treatment characteristic

\begin{tabular}{|c|c|c|c|c|}
\hline Variable & All $(n=85)$ & SIB(-) group & $\mathrm{SIB}(+)$ group $^{\mathrm{a})}$ & p-value \\
\hline Number of patients & 85 & 42 & 43 & \\
\hline Age (yr) & & & & 0.911 \\
\hline$\geq 65$ & $44(51.8)$ & $20(47.6)$ & $22(51.2)$ & \\
\hline$<65$ & $41(48.2)$ & $22(52.4)$ & $21(48.8)$ & \\
\hline Gender & & & & 0.616 \\
\hline Male & $82(96.5)$ & $40(95.2)$ & $42(97.7)$ & \\
\hline Female & $3(3.5)$ & $2(4.8)$ & $1(2.3)$ & \\
\hline Radiotherapy aim & & & & 0.360 \\
\hline Definitive & $80(94.1)$ & $41(97.6)$ & $39(90.7)$ & \\
\hline Salvage & $5(5.9)$ & $1(2.4)$ & $4(9.3)$ & \\
\hline Clinical tumor stage & & & & 0.012 \\
\hline cT1 & $70(82.4)$ & $39(92.9)$ & $31(72.1)$ & \\
\hline cT2 & $15(17.6)$ & $3(7.1)$ & $12(27.9)$ & \\
\hline Anterior commissure involvement & & & & 0.156 \\
\hline Yes & $51(60.0)$ & $22(52.4)$ & $29(67.4)$ & \\
\hline No & $34(40.0)$ & $20(47.6)$ & $14(32.6)$ & \\
\hline Stromal invasion & & & & 0.039 \\
\hline Yes & $18(21.2)$ & $5(11.9)$ & $13(30.2)$ & \\
\hline No & 67 (78.8) & $37(88.1)$ & $30(69.8)$ & \\
\hline Radiotherapy technique & & & & $<0.001$ \\
\hline IMRT & $66(77.6)$ & $23(54.8)$ & $43(100)$ & \\
\hline 3D CRT & $19(22.4)$ & $19(45.2)$ & $0(0)$ & \\
\hline $\mathrm{BED}_{10}$ for the tumor (Gy) & & & & $<0.001$ \\
\hline 84.38 & $37(43.5)$ & $0(0)$ & $37(86.0)$ & \\
\hline$<84.38$ & $48(56.5)$ & $42(100)$ & $6(14.0)$ & \\
\hline
\end{tabular}

$\mathrm{SIB}$, simultaneous integrated boost; $\mathrm{BED}_{10}$, biologically effective dose when $\alpha / \beta$ ratio was presumed to be 10 for early responding tissue; IMRT, intensity-modulated radiotherapy; 3D CRT, three-dimensional conformal radiotherapy.

a) SIB dose for the tumor: $67.5 \mathrm{~Gy}$ (2.5 Gy per fraction) for 37 patients, $64.8 \mathrm{~Gy}$ (2.4 Gy per fraction) for 5 patients, and $62.1 \mathrm{~Gy}$ (2.3 Gy per fraction) for 1 patient.
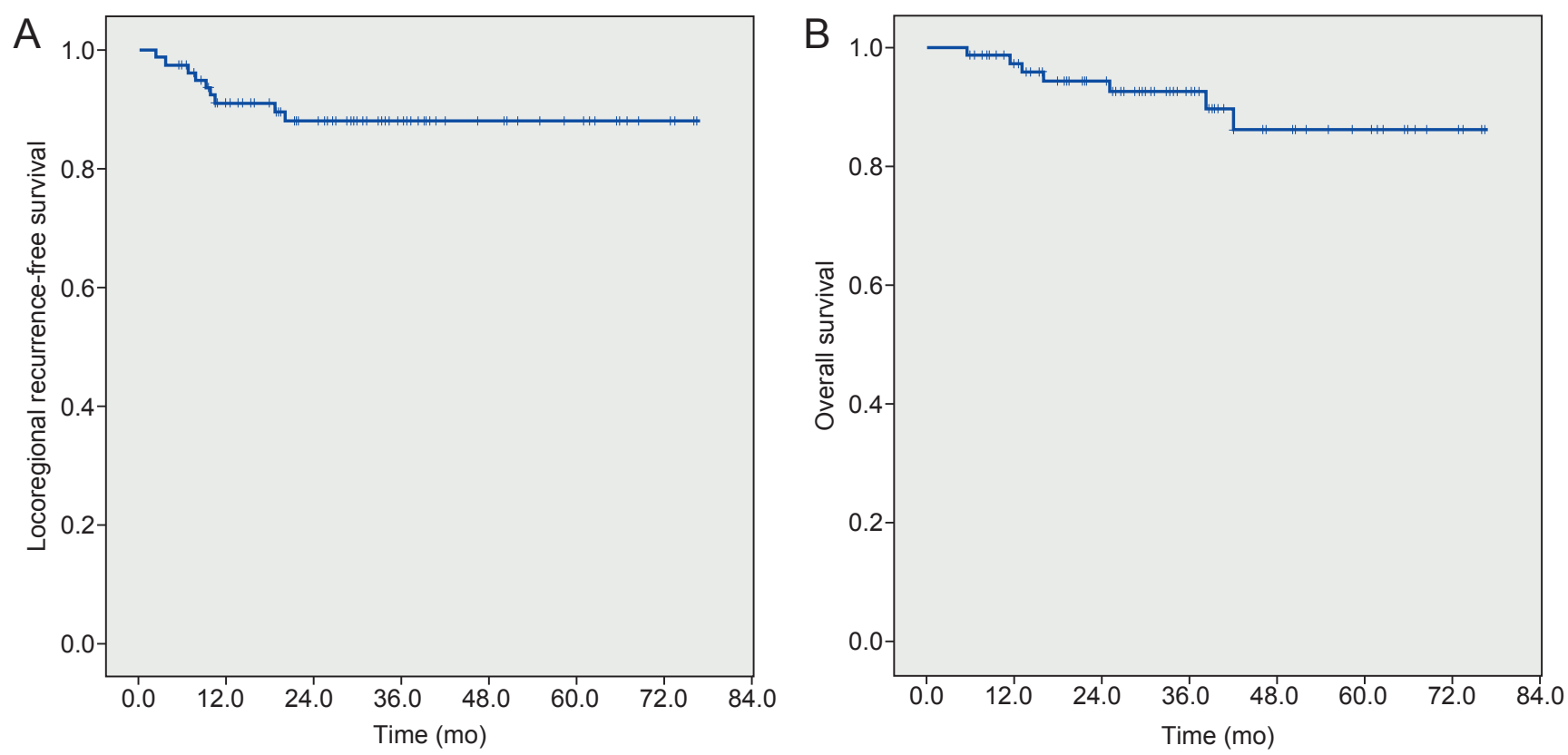

Fig. 1. (A) Locoregional recurrence-free survival rate and (B) overall survival rate. 


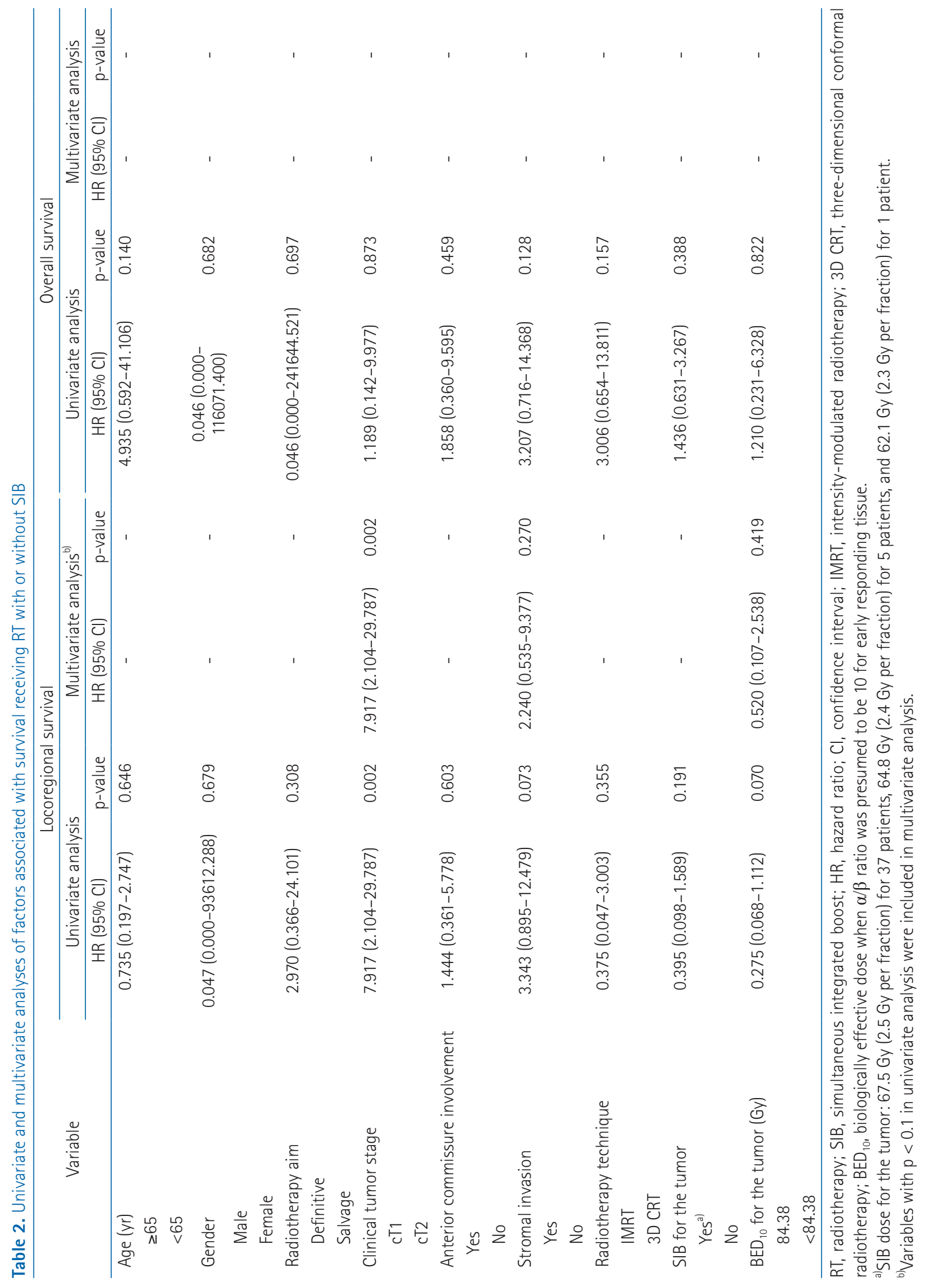




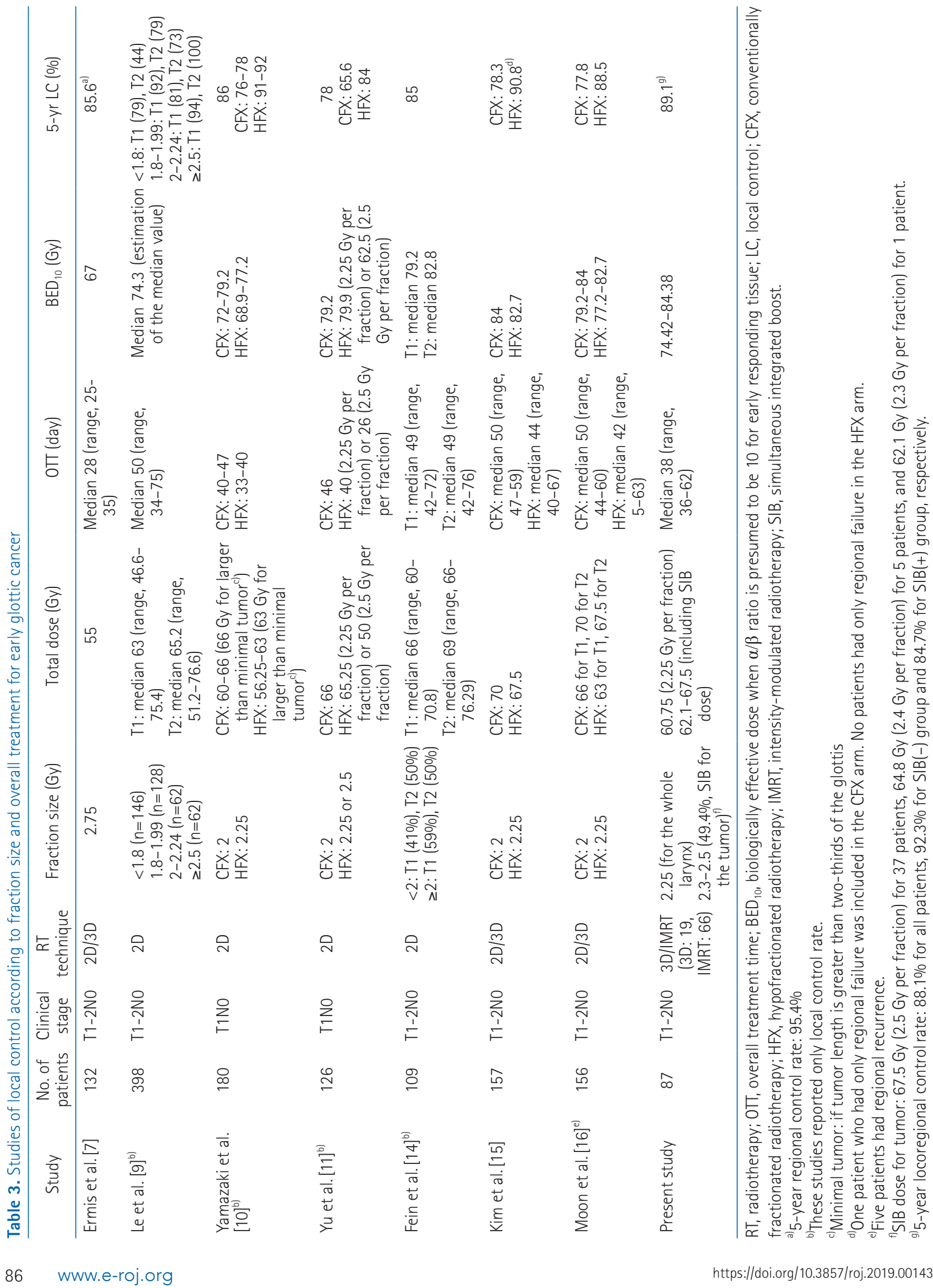


median patient age was 65 years (range, 43 to 86 years). Clinical T1 and T2 diseases were observed in 70 (82.4\%) and 15 (17.6\%) patients, respectively. Eighteen patients (21.2\%) had tumors with stromal invasion and 51 (60.0\%) had tumors with an anterior commissure involvement. For the PTV of gross tumors, 37 patients received a total $\mathrm{BED}_{10}$ of $84.38 \mathrm{~Gy}$, whereas the remaining 48 patients (56.5\%) received a total $\mathrm{BED}_{10}$ of $<84.38 \mathrm{~Gy}_{10}$. The median overall treatment time was 38 days (range, 36 to 62 days). Altogether, 66 patients were treated with IMRT and 19 patients were treated with 3D CRT.

The median follow-up duration was 29.9 months (range, 5.5 to 76.5 months). All patients achieved complete remission after a median of 50 days from the end of RT (range, 14 to 206 days). The 5 -year rates of LRFS and OS were $88.1 \%$ and $86.2 \%$, respectively (Fig. 1A, 1B). Clinical T stage (cT1 vs. cT2) was a statistically significant factor in the univariate and multivariate analyses for LRFS (hazard ratio $[H R]=7.917$; and confidence interval [CI], 2.104-29.787; $p=0.006)$. However, clinical T stage was not associated with OS ( $p=0.881$ ). Age, gender, RT aim (which means recurrent disease or not), anterior commissure involvement, stromal invasion, RT technique, SIB and BED 10 (Gy) for tumor did not affect LRFS and OS (Table 2).

Disease failure was observed in 9 patients (10.6\%), local failure occurred only in 6 patients (7.1\%), locoregional failure occurred in 9 patients (10.6\%), and combined regional and distant failure but without local failure occurred in 1 patient (1.2\%). The median time to disease failure after the end of RT was 9.1 months (range, 2.4 to 20.1 months). Salvage surgery was conducted in seven patients, and the other patients did not receive it. One patient received a second surgery owing to rapid locoregional tumor progression during adjuvant neck RT after the first salvage surgery. Distant metastasis was observed in one patient after salvage surgery. This patient had multiple metastatic lesions in the right cervical and mediastinal lymph nodes, pericardium, both lungs, left adrenal gland, and sacrum; the patient died 7 days after the diagnosis of distant disease failure. Of the 85 patients, 7 patients died. Two events of cancer-specific death (97.6\%) occurred.

Most patients had grade 1 or 2 mucositis and dermatitis during the treatment. Grade 2 chronic laryngeal edema was observed in two patients. No patients experienced acute or chronic toxicities of $\geq$ grade 3 . In the plan analysis of IMRT and 3D CRT for the carotid arteries, the mean of $\mathrm{V}_{35 \mathrm{G} y}$ was $35.4 \%$ (range, $0.0 \%$ to $70.5 \%$ ) for IMRT plans, and 59.8\% (range, $31.9 \%$ to $87.6 \%$ ) for 3D CRT plan. The mean of $\mathrm{V}_{50 \mathrm{~Gy}}$ was $22.2 \%$ (range, $0.0 \%$ to $44.3 \%$ ) for the IMRT plan, and $40.5 \%$ (range, $7.5 \%$ to $73.6 \%$ ) for the 3D CRT plan. Carotid artery dose was more significantly lowered by IMRT than by 3D CRT $\left(\mathrm{V}_{356 \mathrm{y}} \mathrm{p}<\right.$ $\left.0.001 ; V_{506 y} p<0.001\right)$.

\section{Discussion and Conclusion}

In this study, the 5-year rates of LRFS and OS were $88.1 \%$ and $86.2 \%$, respectively. These results are comparable with those of other studies involving HFX [7,9-11,14-16] (Table 3). Le et al. [9] and $Y u$ et al. [11] identified that fraction size was an important prognostic factor for local control. Some studies used a fraction size of $2.25 \mathrm{~Gy}$, which is similar to the fraction size used in our study. Yamazaki et al. [10] reported the outcomes of a randomized trial which analyzed 180 patients with T1 glottic cancer, with a 5 -year local control rate of $77 \%$ for CFX and $92 \%$ for HFX ( $p=0.004)$. The authors concluded that HFX using a radiation dose of 2.25 Gy for a shorter duration showed superior local control than CFX. The KROG-0201 trial [16], a prospective randomized trial, compared HFX (63 Gy in 28 fractions for $\mathrm{T} 1 ; 67.5 \mathrm{~Gy}$ in 30 fractions for T2) with CFX (66 Gy in 33 fractions for T1; 70 Gy in 35 fractions for T2). The local progression-free survival at 5 years was $77.8 \%$ for CFX and $88.5 \%$ for HFX, but the difference was insignificant ( $p=$ 0.213). Those studies reported no significant differences in complication rates between the HFX and CFX arms. Therefore, 2.25 Gy per fraction may be an acceptable fraction size for the whole larynx. Additionally, a study conducted in Yale University analyzed data for EGC patients treated with RT from the National Cancer Database [17]. The authors reported that the use of HFX was associated with better survival than that using CFX, especially for clinical T2 EGC.

Some authors have reported the use of large fraction sizes exceeding 2.5 Gy $[7,8,18]$. These studies delivered a total dose of approximately $50 \mathrm{~Gy}$ in 16 fractions over 3 weeks $[8,18]$ and $55 \mathrm{~Gy}$ in 20 fractions over 4 weeks [7], with median followup durations of 5-7 years. In a study by Ermis et al. [7], the 5 -year local control rate was $85.6 \%$. Gowda et al. [8] reported a 5-year local control rate of 93\%, whereas Cheah et al. [18] reported a 5-year locoregional control rate of $88 \%$. There were acceptable complications, and the cancer-specific survival at 5 years was 95.7\%-97\% in these studies. However, a larger fraction size may cause an increase in adverse complications. After a long follow-up duration of $\geq 5$ years, a higher risk of late complications was found for patients who received RT with a large fraction size of $>3$ Gy [19]. Dinshaw et al. [20] reported that radiation-induced edema occurred in $20 \%$ of the T2 patients treated with a fraction size of $3.3 \mathrm{~Gy}$.

IMRT using SIB has already been widely used in the 


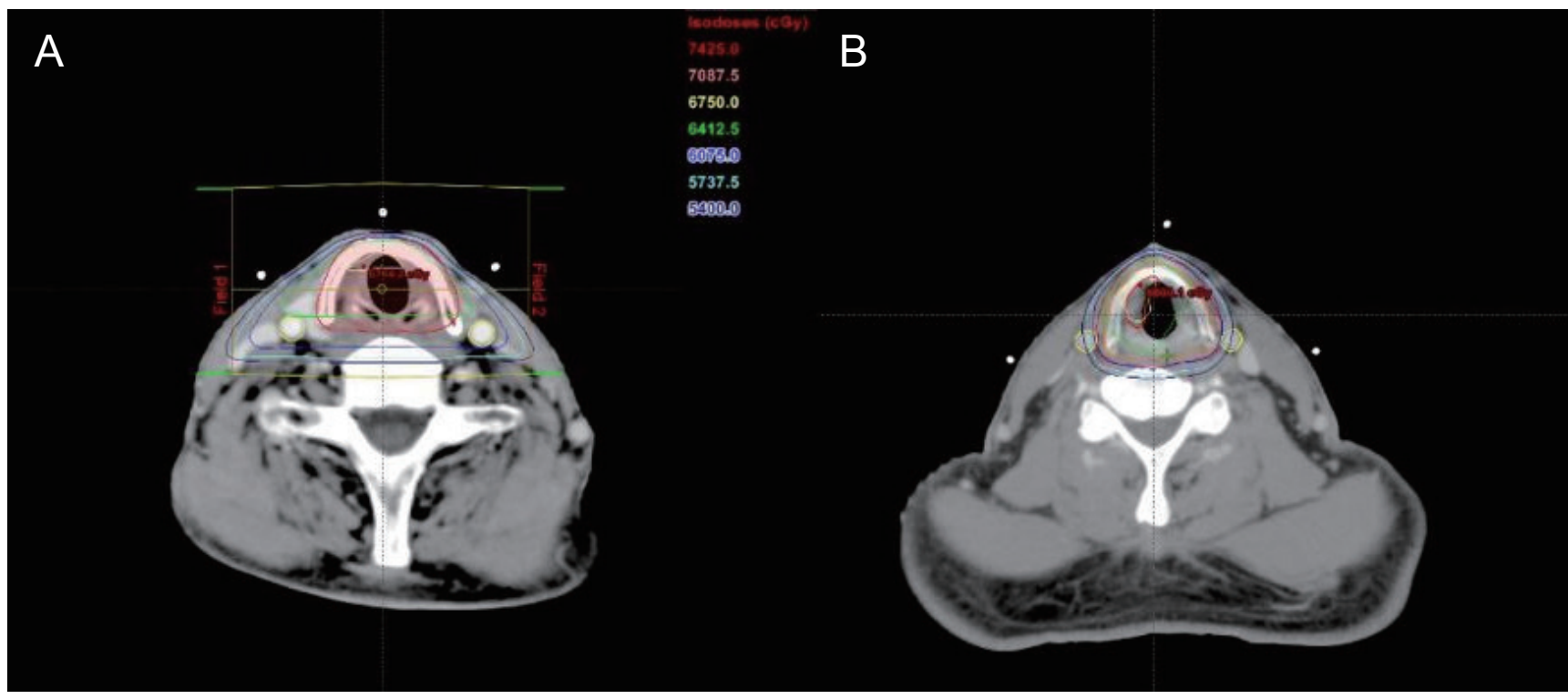

Fig. 2. A comparison of axial slices from the planning computed tomography scan between patients who received three-dimensional conformal radiotherapy (A) and intensity-modulated radiotherapy (B). Both carotid arteries are delineated in yellow.

treatment of head and neck cancer owing to its radiobiologic advantages caused by tumor dose escalation and better target dose distribution with lower organs at risk (OAR) dose [21,22], and Janssen et al. [23] reported positive results of SIB-IMRT for glottic cancer. We used a fraction size of 2.25 Gy to treat EGC, and attempted to achieve a tumor dose escalation of 2.3-2.5 Gy per fraction by SIB-IMRT. Use of SIB technique for the tumor did not affect LRFS ( $p=0.191)$, which suggests that the larynx may be treated in EGC without administration of an additional higher radiation dose of SIB for the tumor.

Anterior commissure $(A C)$ involvement is often considered to be an unfavorable prognostic factor. The AC is connected to the thyroid cartilage, which lacks a tumor barrier [9]. Thus, a tumor with AC involvement may easily penetrate the thyroid cartilage and spread to surrounding tissues. However, there are conflicting reports of $A C$ involvement as a significant prognostic factor $[8,11,15,16,24,25]$. In our study, no significant difference in locoregional control was observed for $A C$ involvement ( $p=0.603)$.

The analyses conducted by Kim et al. [15] and Moon et al. [16] revealed no significant effect of $\mathrm{T} 2$ disease. However, in our study, T2 disease was the only significant predictor of locoregional disease control ( $p=0.002)$. The 10-year local control rate was $82 \%$ and $57 \%$ for $\mathrm{T} 1$ and $\mathrm{T} 2$ diseases $(p=0.00)$ in a study by Dinshaw et al. [20]. As mentioned earlier, SIB was mostly administered to patients who had at least one of the factors including anterior commissure involvement, stromal invasion, or T2 stage at our institution.

The overall treatment time, which is closely connected with the fraction size, is a very important prognostic factor $[5,9,26]$. A larger fraction size facilitates a shorter overall treatment time, which is required to diminish the accelerated repopulation of tumor cells [27]. In an analysis of 91 patients with T1 glottic cancer, Rudoltz et al. [5] found that a dose per fraction of $\geq 2$ Gy provided superior local control than that achieved with a conventional dose per fraction of $<2 \mathrm{~Gy}$. The authors also reported that a prolonged overall treatment time was associated with a decline in local control rate: $100 \%$ within 42 days, 91\% for 43-46 days, 74\% for 47-50 days, 65\% for 51-54 days, and 50\% for 55-66 days ( $p=0.0001$ ). Onimaru et al. [26] used a cutoff of 46 days for the overall treatment time of T1 glottic cancer, which showed a significant difference before and after the cutoff ( $p=0.0349$ ). In our study, the treatment of 80 patients was completed within 46 days, even if there were 7 cases of locoregional recurrences among them. However, the results of this study may not be comparable because of the inclusion of $\mathrm{T} 2$ and recurrent diseases.

RT may increase the risk of cerebrovascular accidents (CVA) [13]. Although there was no patient in the present study who had CVA after RT, a higher risk of fatal CVA after RT was reported in patients receiving $\mathrm{RT}$ than the risk associated with surgery for EGC in the Surveillance, Epidemiology, and End Results (SEER) database (2.8\% vs. 1.5\%, $p=0.037$ ) [28] Other SEER analyses revealed an increased incidence of CVA 
in elderly EGC patients at approximately $8 \%$ in patients receiving $R T$ than surgery $[29,30]$. There are a variety of ways to prevent carotid artery delineation and dose constraint [31]. Most studies suggest a distance of 1-2.5 cm from PTV as the superior and inferior extent of the carotid artery [3133]. In our study, the carotid artery was considered to be the single OAR. We used a $1.5-\mathrm{cm}$ extension craniocaudally from PTV, which is similar to the procedure implemented in studies by Kim et al. [33] and Matthiesen et al. [34]. Although the planning procedures for IMRT in our study were performed without the intent of carotid artery preservation, IMRT showed lower $V_{356 y}$ and $V_{506 y}$ of the carotid artery than did 3D CRT ( $p$ $<0.001)$. These dosimetric advantages of IMRT may not be inferior to those reported by Berwouts et al. [12] and Janssen et al. [23]. Consequently, IMRT may decrease the dose for OARs exposed to CFX (Fig. 2). Our study included clinical experiences for IMRT, which is valuable because most studies on HFX were implemented using 2D/3D technique, and many IMRT studies were focused on the dosimetric aspect of EGC $[7,9-11,14$ $16,31]$.

There are some limitations to our study because of its retrospective design. The number of patients was relatively small, and the median follow-up duration was short. Further comprehensive studies with long follow-up period are needed to confirm the results of this study.

In conclusion, HFX resulted in a high locoregional disease control rate and overall survival rate. A fraction size of 2.25 Gy provided good local control, regardless of the use of SIB. Furthermore, IMRT may reduce toxicity by sparing the carotid artery.

\section{Conflict of Interest}

No potential conflict of interest relevant to this article was reported.

\section{References}

1. Mendenhall WM, Amdur RJ, Morris CG, Hinerman RW. T1T2NO squamous cell carcinoma of the glottic larynx treated with radiation therapy. J Clin Oncol 2001;19:4029-36.

2. Lim YJ, Wu HG, Kwon TK, et al. Long-term outcome of definitive radiotherapy for early glottic cancer: prognostic factors and patterns of local failure. Cancer Res Treat 2015;47:862-70.

3. Smee RI, Meagher NS, Williams JR, Broadley K, Bridger GP. Role of radiotherapy in early glottic carcinoma. Head Neck
2010;32:850-9.

4. Mendenhall WM, Parsons JT, Million RR, Fletcher GH. T1-T2 squamous cell carcinoma of the glottic larynx treated with radiation therapy: relationship of dose-fractionation factors to local control and complications. Int J Radiat Oncol Biol Phys 1988;15:1267-73.

5. Rudoltz MS, Benammar A, Mohiuddin M. Prognostic factors for local control and survival in T1 squamous cell carcinoma of the glottis. Int J Radiat Oncol Biol Phys 1993;26:767-72.

6. Schwaibold F, Scariato A, Nunno M, et al. The effect of fraction size on control of early glottic cancer. Int J Radiat Oncol Biol Phys 1988;14:451-4.

7. Ermis E, Teo M, Dyker KE, Fosker C, Sen M, Prestwich RJ. Definitive hypofractionated radiotherapy for early glottic carcinoma: experience of 55Gy in 20 fractions. Radiat Oncol 2015;10:203.

8. Gowda RV, Henk JM, Mais KL, Sykes AJ, Swindell R, Slevin NJ. Three weeks radiotherapy for T1 glottic cancer: the Christie and Royal Marsden Hospital Experience. Radiother Oncol 2003;68:105-11.

9. Le QT, Fu KK, Kroll S, et al. Influence of fraction size, total dose, and overall time on local control of T1-T2 glottic carcinoma. Int J Radiat Oncol Biol Phys 1997;39:115-26.

10. Yamazaki H, Nishiyama K, Tanaka E, Koizumi M, Chatani M. Radiotherapy for early glottic carcinoma (T1NOMO): results of prospective randomized study of radiation fraction size and overall treatment time. Int J Radiat Oncol Biol Phys 2006;64:77-82.

11. Yu E, Shenouda G, Beaudet MP, Black MJ. Impact of radiation therapy fraction size on local control of early glottic carcinoma. Int J Radiat Oncol Biol Phys 1997;37:587-91.

12. Berwouts $D$, Swimberghe $M$, Duprez $F$, et al. Intensitymodulated radiotherapy for early-stage glottic cancer. Head Neck 2016;38 Suppl 1:E179-84.

13. Martin JD, Buckley AR, Graeb D, Walman B, Salvian A, Hay JH. Carotid artery stenosis in asymptomatic patients who have received unilateral head-and-neck irradiation. Int J Radiat Oncol Biol Phys 2005;63:1197-205.

14. Fein DA, Lee WR, Hanlon AL, Ridge JA, Curran WJ, Coia LR. Do overall treatment time, field size, and treatment energy influence local control of T1-T2 squamous cell carcinomas of the glottic larynx? Int J Radiat Oncol Biol Phys 1996;34:82331.

15. Kim TG, Ahn YC, Nam HR, et al. Definitive radiation therapy for early glottic cancer: experience of two fractionation schedules. Clin Exp Otorhinolaryngol 2012;5:94-100.

16. Moon $\mathrm{SH}$, Cho KH, Chung EJ, et al. A prospective randomized 
trial comparing hypofractionation with conventional fractionation radiotherapy for T1-2 glottic squamous cell carcinomas: results of a Korean Radiation Oncology Group (KR0G-0201) study. Radiother Oncol 2014;110:98-103.

17. Bledsoe TJ, Park HS, Stahl JM, et al. Hypofractionated radiotherapy for patients with early-stage glottic cancer: patterns of care and survival. J Natl Cancer Inst 2017;109:djx042. https://doi.org/10.1093/jnci/djx042

18. Cheah NL, Lupton S, Marshall A, Hartley A, Glaholm J. Outcome of T1NOMO squamous cell carcinoma of the larynx treated with short-course radiotherapy to a total dose of 50 Gy in 16 fractions: the Birmingham experience. Clin Oncol (R Coll Radiol) 2009;21:494-501.

19. van der Voet JC, Keus RB, Hart AA, Hilgers FJ, Bartelink $H$. The impact of treatment time and smoking on local control and complications in T1 glottic cancer. Int J Radiat Oncol Biol Phys 1998:42:247-55.

20. Dinshaw KA, Sharma V, Agarwal JP, Ghosh S, Havaldar R. Radiation therapy in T1-T2 glottic carcinoma: influence of various treatment parameters on local control/complications. Int J Radiat Oncol Biol Phys 2000;48:723-35.

21. Leclerc $M$, Maingon $P$, Hamoir $M$, et al. A dose escalation study with intensity modulated radiation therapy (IMRT) in T2NO, T2N1, T3NO squamous cell carcinomas (SCC) of the oropharynx, larynx and hypopharynx using a simultaneous integrated boost (SIB) approach. Radiother Oncol 2013;106:333-40.

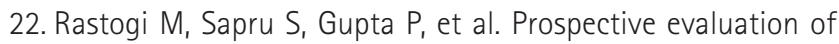
intensity modulated radiation therapy with simultaneous integrated boost (IMRT-SIB) in head and neck squamous cell carcinoma in patients not suitable for chemo-radiotherapy. Oral Oncol 2017;67:10-6.

23. Janssen S, Glanzmann C, Huber G, Studer G. Risk-adapted partial larynx and/or carotid artery sparing modulated radiation therapy of glottic cancer. Radiat Oncol 2014;9:136.

24. Laskar SG, Baijal G, Murthy V, et al. Hypofractionated radiotherapy for $\mathrm{T} 1 \mathrm{NOMO}$ glottic cancer: retrospective analysis of two different cohorts of dose-fractionation schedules from a single institution. Clin Oncol (R Coll Radiol) 2012;24:e180-6.
25. Olszewski SJ, Vaeth JM, Green JP, Schroeder AF, Chauser B. The influence of field size, treatment modality, commissure involvement and histology in the treatment of early vocal cord cancer with irradiation. Int J Radiat Oncol Biol Phys 1985;11:1333-7.

26. Onimaru R, Hasegawa $M$, Yasuda $K$, et al. Radiotherapy for glottic T1NO carcinoma with slight hypofractionation and standard overall treatment time: importance of overall treatment time. Jpn J Clin Oncol 2011;41:103-9.

27. Chera BS, Amdur RJ, Morris CG, Kirwan JM, Mendenhall WM. T1NO to T2NO squamous cell carcinoma of the glottic larynx treated with definitive radiotherapy. Int J Radiat Oncol Biol Phys 2010;78:461-6.

28. Swisher-McClure S, Mitra N, Lin A, et al. Risk of fatal cerebrovascular accidents after external beam radiation therapy for early-stage glottic laryngeal cancer. Head Neck 2014;36:611-6.

29. Hong JC, Kruser TJ, Gondi V, et al. Risk of cerebrovascular events in elderly patients after radiation therapy versus surgery for early-stage glottic cancer. Int J Radiat Oncol Biol Phys 2013;87:290-6.

30. Smith GL, Smith BD, Buchholz TA, et al. Cerebrovascular disease risk in older head and neck cancer patients after radiotherapy. J Clin Oncol 2008;26:5119-25.

31. Gujral DM, Long M, Roe JW, Harrington KJ, Nutting CM. Standardisation of target volume delineation for carotidsparing intensity-modulated radiotherapy in early glottis cancer. Clin Oncol (R Coll Radiol) 2017;29:42-50.

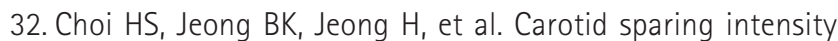
modulated radiotherapy on early glottic cancer: preliminary study. Radiat Oncol J 2016;34:26-33.

33. Kim YS, Lee J, Park JI, Sung W, Lee SM, Kim GE. Volumetric modulated arc therapy for carotid sparing in the management of early glottic cancer. Radiat Oncol J 2016;34:18-25.

34. Matthiesen $C_{1}$ Herman $T_{\text {, Sing }} H_{\text {, et al. Dosimetric and }}$ radiobiologic comparison of $3 \mathrm{D}$ conformal, IMRT, VMAT and proton therapy for the treatment of early-stage glottic cancer. J Med Imaging Radiat Oncol 2015;59:221-8. 